\title{
Alteration in lymphocyte responses, cytokine and chemokine profiles in chickens infected with genotype VII and VIII velogenic Newcastle disease virus
}

\begin{abstract}
Newcastle disease (ND) is a highly contagious avian disease and one of the major causes of economic losses in the poultry industry. The emergence of virulent NDV genotypes and repeated outbreaks of NDV in vaccinated chickens have raised the need for fundamental studies on the virus-host interactions. In this study, the profiles of B and T lymphocytes and macrophages and differential expression of 26 immune-related genes in the spleen of specific-pathogen-free (SPF) chickens, infected with either the velogenic genotype VII NDV strain IBS002 or the genotype VIII NDV strain AF2240, were evaluated. A significant reduction in $\mathrm{T}$ lymphocyte population and an increase in the infiltration of IgM+ B cells and KUL01+ macrophages were detected in the infected spleens at 1, 3 and 4 days post-infection (dpi) $(\mathrm{P}<0.05)$. The gene expression profiles showed an up-regulation of CCLi3, CXCLi1, CXCLi2 (IL-8), IFN- $\gamma$, IL-12 $\alpha$, IL-18, IL-1 $\beta$, IL-6, iNOS, TLR7, MHCI, IL-17F and TNFSF13B (P < 0.05). However, these two genotypes showed different cytokine expression patterns and viral load. IBS002 showed higher viral load than AF2240 in spleen at 3 and 4 dpi and caused a more rapid up-regulation of CXCLi2, IFN- $\gamma$, IL-12 $\alpha$, IL-18, IL-1 $\beta$, iNOS and IL-10 at 3 dpi. Meanwhile, the expression levels of CCLI3, CXCLi1, IFN- $\gamma$, IL-12 $\alpha$, IL$1 \beta$ and iNOS genes were significantly higher in AF2240 at 4 dpi. In addition, the expression levels of IL-10 were significantly higher in the IBS002-infected chickens at 3 and 4 dpi. Hence, infection with velogenic genotype VII and VIII NDV induced different viral load and production of cytokines and chemokines associated with inflammatory reactions.
\end{abstract}

Keyword: Newcastle disease virus; GeXP; Cytokine; Gene expression 